\title{
A NOTE ON THE CHARACTERISATION OF EIGENCURVES FOR CERTAIN TWO PARAMETER EIGENVALUE PROBLEMS IN ORDINARY DIFFERENTIAL EQUATIONS
}

\author{
by PATRICK J. BROWNE† and B. D. SLEEMAN
}

(Received 25 July, 1991)

\begin{abstract}
1. Introduction We are interested in two parameter eigenvalue problems of the form

$$
-y^{\prime \prime}(x)+q(x) y(x)=(\lambda s(x)-\mu) y(x), \quad 0 \leq x \leq 1,
$$

subject to Dirichlet boundary conditions

$$
y(0)=y(1)=0
$$

The weight function $s$ and the potential $q$ will both be assumed to lie in $L^{2}[0,1]$. The problem (1.1), (1.2) generates eigencurves

$$
\mu^{1}(\lambda)>\mu^{2}(\lambda)>\ldots
$$

in the sense that for any fixed $\lambda, \mu^{n}(\lambda)$ is the $n$th eigenvalue $\mu$, (according to oscillation indexing) of (1.1), (1.2). These curves are in fact analytic functions of $\lambda$ and have been the object of considerable study in recent years. The survey paper [1] provides background in this area and itemises properties of eigencurves.

While much is known about the eigencurves of (1.1), (1.2) when $s$ and $q$ are given, questions of an inverse nature associated with such problems have been thus far little studied and seem to be intrinsically difficult. Perhaps the most basic problem in the development of an inverse spectral theory for $(1.1),(1.2)$ is that of characterising those families of analytic curves which arise as the eigencurves generated by some weight and potential, $s$ and $q$, via (1.1), (1.2). The aim of this note is to give a partial resolution to this question.

The corresponding problem for one parameter equations has been completely resolved. Here one is concerned with a Dirichlet eigenvalue problem

$$
-y^{\prime \prime}(x)+q(x) y(x)=\rho y(x), \quad 0 \leq x \leq 1, \quad y(0)=y(1)=0 .
$$

The eigenvalues $\rho^{n}$ of this problem are strictly increasing and must necessarily be of the form

$$
\rho^{n}=n^{2} \pi^{2}+[q]+a_{n}, \quad n \geq 1,
$$

where $[q]=\int_{0}^{1} q(x) d x$ and $\sum a_{n}^{2}<\infty$. Further any strictly increasing sequence $\rho^{n}$ satisfying

$$
\rho^{n}=n^{2} \pi^{2}+\alpha+a_{n}, \quad \alpha \in R, \quad \sum a_{n}^{2}<\infty,
$$

must be the Dirichlet eigenvalue sequence for some potential $q \in L^{2}[0,1]$ and, indeed, for a unique 'even' $q$ where we shall call a function $f$ even if

$$
f(x)=f(1-x), \quad 0 \leq x \leq 1 .
$$

$†$ Research supported in part by a grant from the NSERC of Canada

Glasgow Math. J. 35 (1993) 63-67. 
These results are given by Pöschel and Trubowitz in [2] where there is also a formula for finding the even potential $q$ corresponding to (1.4). Specifically, we define

$$
\begin{gathered}
\Pi=\prod_{j>i \geq 1}\left(\frac{\rho^{i}-\alpha-j^{2} \pi^{2}}{\rho^{i}-\rho^{j}} \frac{i^{2} \pi^{2}-\rho^{j}+\alpha}{i^{2} \pi^{2}-j^{2} \pi^{2}}\right) \\
\omega_{i j}(x)=\frac{\rho^{i}-\alpha-i^{2} \pi^{2}}{\rho^{i}-\alpha-j^{2} \pi^{2}}\left[\cos \left(\sqrt{\rho^{i}-\alpha}\right) x+\frac{(-1)^{i}-\cos \sqrt{\rho^{i}-\alpha}}{\sin \sqrt{\rho^{i}-\alpha}} \sin \left(\sqrt{\rho^{i}-\alpha}\right) x, \frac{\sin \pi j x}{\pi j}\right],
\end{gathered}
$$

where $[\cdot, \cdot]$ denotes the Wronskian, and $D$ to be the infinite determinant

$$
D(x)=\operatorname{det}\left(\omega_{i_{j}}(x)\right)
$$

Then

$$
q(x)=-2 \frac{d^{2}}{d x^{2}} \log (\Pi D(x))+\alpha
$$

is the required unique even $q$. Full details of these constructions are given in [2, Chapter 6].

It will be necessary for us to consider a subclass of two parameter problems and accordingly we have:

Definition. The problem (1.1), (1.2) is said to be evenly generated if there are even functions $Q, S \in L^{2}[0,1]$ so that the problem

$$
\begin{gathered}
-y^{\prime \prime}(x)+Q(x) y(x)=(\lambda S(x)-\mu) y(x), \quad 0 \leq x \leq 1, \\
y(0)=y(1)=0
\end{gathered}
$$

has the same eigencurves $\mu^{n}(\lambda), n \geq 1$, as (1.1), (1.2). We call $Q$ (respectively, $S$ ) the equivalent even potential (respectively, weight).

It is clear that if $q$ and $s$ are even at the outset then (1.1), (1.2) is evenly generated and also that if $s$ is constant then (1.1), (1.2) is evenly generated.

Our results, presented in the next section, will characterise the evenly generated problems in terms of their eigencurves and will also characterise those families $\left\{\mu^{n}(\lambda)\right\}$ of analytic curves which arise as eigencurves of evenly generated problems.

Each of us is grateful to the other's institution for hospitality extended during recent reciprocal visits.

2. Results. We begin by noting that by virtue of (1.3), the eigencurves of (1.1), (1.2) must satisfy

$$
\mu^{n}(\lambda)=-n^{2} \pi^{2}-[q]+\lambda[s]+r_{n}(\lambda), \quad n \geq 1,
$$

where $\sum r_{n}(\lambda)^{2}<\infty, \lambda \in \mathbf{R}$. Further when (1.1), (1.2) is evenly generated with equivalent potential, weight $Q, S$ we also have

$$
[q]=[Q] \text { and }[s]=[S]
$$

For curves of the form

$$
\mu^{n}(\lambda)=-n^{2} \pi^{2}-\alpha+\lambda \beta+r_{n}(\lambda), \quad n \geq 1, \quad \sum r_{n}^{2}(\lambda)<\infty
$$


with $\mu^{1}(\lambda)>\mu^{2}(\lambda)>\ldots$, we note that $-\mu^{n}(\lambda)$ is, for each fixed $\lambda \in \mathbf{R}$, of the form (1.4) and accordingly we can define the infinite product (1.5) and the infinite determinant (1.6) now as functions of both $\lambda$ and $x$. We denote these constructions by $\Pi(\lambda)$ and $D(\lambda, x)$.

THEOREM 2.1. Suppose (1.1), (1.2) is evenly generated with equivalent even potential and weight $Q$ and $S$ respectively. Then

$$
\frac{d^{2}}{d x^{2}} \log \left(\frac{\Pi(\lambda) D(\lambda, x)}{\Pi(0) D(0, x)}\right)=\frac{1}{2} \lambda\{S(x)-[s]\}, \quad 0 \leq x \leq 1, \quad \lambda \in \mathbf{R} .
$$

Note that (2.3) is in essence a condition on the residual terms $r_{n}(\lambda)$ in (2.1).

Proof. We apply the one parameter result (1.7) at $\lambda=0$ and at arbitrary $\lambda$ to obtain

$$
\begin{gathered}
Q(x)=-2 \frac{d^{2}}{d x^{2}}(\log \Pi(0) D(0, x))+[q], \\
Q(x)-\lambda S(x)=-2 \frac{d^{2}}{d x^{2}}(\log \Pi(\lambda) D(\lambda, x))+[q]-\lambda[s],
\end{gathered}
$$

from which the result follows immediately.

THEOREM 2.2. Suppose the eigencurves of (1.1), (1.2) satisfy (2.3) for some even function $S$. Then (1.1), (1.2) is evenly generated, $S$ is an equivalent even weight and $Q$, given by (2.4), is an equivalent even potential.

Proof. We know, again from (1.7), that (2.4) gives the unique even function $Q(x)$ whose Dirichlet eigenvalues are $\left\{-\mu^{n}(0)\right\}$ and that the right hand side of (2.5) gives the unique even function $H(\lambda, x)$ whose Dirichlet eigenvalues are $\left\{-\mu^{n}(\lambda)\right\}$. The condition (2.3) which we are now assuming, guarantees that

$$
H(x, \lambda)=Q(x)-\lambda S(x)
$$

and so (1.1), (1.2) is evenly generated with $Q, S$ being equivalent even potential and weight.

The upshot of these two theorems is a complete characterization, in terms of the residuals $r_{n}(\lambda)$ of evenly generated problems. We can now go one step further and shed some light on the general question, "which families $\mu^{n}(\lambda)$ of analytic curves are eigencurves of problems (1.1), (1.2)?"

THEOREM 2.3. Suppose we are given a sequence of analytic curves $\mu^{1}\left(\lambda>\mu^{2}(\lambda)>\ldots\right.$ of the form (2.2) for some $\alpha, \beta \in \mathbf{R}$. Then there are the eigencurves of an evenly generated problem (1.1), (1.2) if and only if

$$
\frac{d^{2}}{d x^{2}} \log \left(\frac{\Pi(\lambda) D(\lambda, x)}{\Pi(0) D(0, x)}\right)=\frac{1}{2} \lambda\{S(x)-\beta\}, \quad 0 \leq x \leq 1, \quad \lambda \in \mathbf{R},
$$

for some even function $S$ which will be the weight function in (1.1). The potential $Q$ will be given by

$$
Q(x)=-2 \frac{d^{2}}{d x^{2}}(\log \Pi(0) D(0, x))+\alpha
$$


and further we have

$$
[S]=\beta, \quad[Q]=\alpha .
$$

Proof. The proof is much as before. For the sufficiency of the condition, the properties of $\mu^{n}(\lambda)$ ensure the existence of $H(x, \lambda)$ which is even and has $\left\{-\mu^{n}(\lambda)\right\}$ as its set of Dirichlet eigenvalues. Further $[H(x, \lambda)]=\alpha-\lambda \beta$. The condition of the theorem then forces

$$
H(x, \lambda)=Q(x)-\lambda S(x)
$$

and the result follows. The necessity is clear.

We can also study allied questions concerning the existence of problems (1.1), (1.2) with a given weight, whose eigencurves pass through given data points. Specifically, we have

THEOREM 2.4. Suppose we are given an even function $S(x)$ and two strictly decreasing sequences

$$
\begin{aligned}
& \mu_{0}^{n}=-n^{2} \pi^{2}-\alpha+r_{n, 0}, \quad \sum\left(r_{n, 0}\right)^{2}<\infty \\
& \mu_{1}^{n}=-n^{2} \pi^{2}-\alpha+[s]+r_{n, 1}, \quad \sum\left(r_{n, 1}\right)^{2}<\infty .
\end{aligned}
$$

We use $\left\{-\mu_{0}^{n}\right\},\left\{-\mu_{1}^{n}\right\}$ to construct infinite products and determinants $\Pi(i), D(i, x)$, $i=0,1$ as in (1.5), (1.6). Then there is an even potential $Q(x)$ so that the eigencurves of (1.1), (1.2) with $q=Q, s=S$ satisfy

$$
\mu^{n}(0)=\mu_{0}^{n}, \mu^{n}(1)=\mu_{1}^{n}, \quad n \geq 1,
$$

if, and only if

$$
\frac{d^{2}}{d x^{2}} \log \left(\frac{\Pi(1) D(1, x)}{\Pi(0) D(0, x)}\right)=\frac{1}{2}\{S(x)-[S]\} .
$$

Proof. Again we need only discuss the sufficiency. There are unique even potentials $Q, H$ whose Dirichlet eigenvalues are $\left\{-\mu_{0}^{n}\right\}$ and $\left\{-\mu_{1}^{n}\right\}$ respectively and the condition implies that $H=Q-S$ and so the result follows.

Obvious extensions of this theorem can be formulated to cover cases in which $S(x)$ and a finite or even countable number of data sequences $\mu_{i}^{n}$ are given and for which we seek a potential so that the eigencurves of $(1.1),(1.2)$ satisfy $\mu^{n}\left(\lambda_{i}\right)=\mu_{i}^{n}$ for some pre-assigned values $\lambda_{i}$.

Further extensions are possible when data on horizontal lines are given. Here we assume we are given $s \in C^{2}[0,1]$ with $s(x)>0,0 \leq x \leq 1$. We then apply the Louiville transformation to the standard problem (1.1), (1.2). Specifically, we use the change of independent variable

$$
z=\frac{1}{k} \int_{0}^{x} s^{1 / 2}(t) d t, \quad k=\int_{0}^{1} s^{1 / 2}(t) d t
$$


in conjunction with

$$
u=s^{1 / 4} y
$$

to obtain the Dirichlet problem (in $z$ )

$$
-\ddot{u}+k^{2}\left(\frac{q+\mu}{s}+\frac{\left(s^{1 / 4}\right) \cdot *}{s^{1 / 4}}\right) u=\lambda k^{2} u, \quad u(0)=u(1)=0,
$$

where $\cdot \equiv \frac{d}{d z}$. Our theorems above can now be adapted to this problem in terms of given curves $\lambda^{n}(\mu)$ or given data $\lambda_{i}^{n}, i=0,1$. The details are easy to supply.

If we continue to asume $s \in C^{2}[0,1]$ and take $m<\min _{[0,1]} s(x)$, we can consider the line $\mu=\lambda m$. With $\mu=\lambda m$ in (1.1) we obtain

$$
-y^{\prime \prime}+q y=\lambda(s-m),
$$

to which we can again apply the Louiville transformation now with

$$
z=\frac{1}{k} \int_{0}^{x}(s-m)^{1 / 2}, \quad k=\int_{0}^{1}(s-m)^{1 / 2}, \quad u=(s-m)^{1 / 4} y
$$

to obtain

$$
-\ddot{u}+k^{2}\left(\frac{q}{s-m}+\frac{\left((s-m)^{1 / 4}\right)^{\cdots}}{(s-m)^{1 / 4}}\right) u=k^{2} \lambda u .
$$

This will lead to characterizations of the intersections of the eigencurves of (1.1), (1.2) with lines through the origin in the $(\lambda, \mu)$ plane.

\section{REFERENCES}

1. P. J. Browne, Two parameter eigencurve theory, in Ordinary and Partial Differential Equations Vol. II, Pitman Research Notes in Mathematics No. 216, (Longman, 1989), 52-60.

2. J. Poschel and E. Trubowitz, Inverse spectral theory, (Academic Press, 1987).

Department of Mathematics and Statistics

UNIVERSITY OF CALGARY

Calgary Alberta

CANADA T2N 1N4

Department of Mathematics and Computer Science

UNIVERSITY OF DUNDEE

DUNDEE DD1 4HN

SCOTLAND 